\title{
Risk Factors for Postoperative Rebleeding and Short-Term Prognosis of Spontaneous Cerebellar Hemorrhage
}

\section{Jun Shen (iD) \\ Xuefei Shao* \\ Ruixiang $\mathrm{Ge} \mathbb{D}^{*}$ \\ Guangfu Di \\ Xiaochun Jiang}

Department of Neurosurgery, The First Affiliated Hospital (YijiShan Hospital) of Wannan Medical College, Wuhu City, Anhui, 24100I, People's Republic of China

*These authors contributed equally to this work
Correspondence: Xiaochun Jiang Department of Neurosurgery, The First Affiliated Hospital (YijiShan Hospital) of Wannan Medical College, Wuhu City, Anhui, 24I00I, People's Republic of China Tel/Fax +8605535739595

Email jiangxiaochun200I@hotmail.com
Purpose: Postoperative rebleeding (PRB) is one of the most severe complications after hematoma evacuation of spontaneous intracerebral hemorrhage (ICH). PRB has been proven to be an independent risk factor for poor prognosis. Previous studies have shown that spot sign and blend sign are independent risk factors for PRB of spontaneous ICH. However, the risk factors for PRB of spontaneous cerebellar hemorrhage ( $\mathrm{SCH}$ ) have not been elucidated. The aim of the present study was to investigate the possible risk factors for PRB and shortterm prognosis of patients with $\mathrm{SCH}$.

Patients and Methods: This study identified 62 patients with $\mathrm{SCH}$ who underwent hematoma evacuation in our department. Risk factors for PRB and short-term prognosis were identified by a univariable logistic regression model, and predictors with a $P$ value of less than 0.05 were included in the multivariable logistic regression model to identify independent predictors. A receiver operating characteristic (ROC) curve was created to test the sensitivity and specificity of independent risk factors.

Results: Hematoma volume was the only independent predictor of PRB (OR=15.14, 95\% $\mathrm{CI}=1.08-213.1, P=0.044)$. The sensitivity and specificity of hematoma volume to PRB were $63.6 \%$ and $89.7 \%$, respectively, and the cutoff value of hematoma volume was $>29.3 \mathrm{~mL}$. GCS score $\leq 8(\mathrm{OR}=5.131,95 \% \mathrm{CI}=1.030-25.554, P=0.046)$ and $\mathrm{PRB}(\mathrm{OR}=13.17,95 \%$ $\mathrm{CI}=1.316-131.798, P=0.028)$ were independent risk factors for poor prognosis of patients with SCH. The sensitivity and specificity of the GCS score to poor prognosis were $66.7 \%$ and $86.2 \%$, respectively. The sensitivity and specificity of the PRB to poor prognosis were $36.4 \%$ and $96.6 \%$, respectively.

Conclusion: Hematoma volume is likely to be a strong predictor of PRB among patients with SCH. GCS scores $\leq 8$ on arrival and PRB were significant predictors of short-term poor outcome.

Keywords: Glasgow Coma Scale, modified Rankin Scale, postoperative rebleeding, spontaneous cerebellar hemorrhage, spontaneous intracerebral hemorrhage

\section{Introduction}

Spontaneous intracerebral hemorrhage (ICH) affects approximately 2 million persons worldwide annually. ICH is a relatively common subtype of stroke, and it is associated with high mortality and functional disability rates. ${ }^{1,2}$ Spontaneous cerebellar hemorrhage $(\mathrm{SCH})$ accounts for $10 \%$ of all ICHs and leads to more devastating consequences, with a 30 -day mortality rate ranging from $30 \%$ to $50 \%$. $^{3,4}$ Hematoma evacuation for SCH may prevent secondary brain injury, brainstem compression, 
fourth ventricle blockage, obstructive hydrocephalus, and herniation. $^{5-9}$ Although it is unclear whether surgical treatment of spontaneous ICH can improve the prognosis of patients compared with conservative treatment, a reduction in surgical complications is considered to improve patient outcomes. $^{8-11}$

Postoperative rebleeding (PRB) is one of the most severe complications after hematoma evacuation for spontaneous ICH. PRB has been proven to significantly increase the mortality of ICH patients and is an independent risk factor for poor prognosis. ${ }^{12}$ Previous studies have shown that spot sign and blend sign are independent risk factors for PRB of spontaneous ICH. ${ }^{13-15}$ However, the risk factors for PRB of SCH have not been fully elucidated. In the present study, we aimed to investigate the possible risk factors for PRB and the short-term prognosis of patients with $\mathrm{SCH}$.

\section{Materials and methods}

\section{Patients and Methods}

\section{Exclusion Criteria}

In this retrospective cohort study, $69 \mathrm{SCH}$ patients who underwent surgical evacuation at the Department of Neurosurgery at The First Affiliated Hospital (YiJiShan Hospital) of Wannan Medical College during 2014-2020 were analyzed. The exclusion criteria included: (1) patients younger than 18 years old at the time of diagnosis; (2) SCH secondary to tumor or vascular anomalies (aneurysms, arteriovenous malformations, moyamoya disease, and arteriovenous fistulas); (3) patients who died within 24 hours after the initial operation or died before the postoperative computed tomography (CT) examination was performed; and (4) patients with missing data. Patients with coagulopathy were not excluded.

\section{The Surgical Indications and Procedure}

The surgical indications were hematoma $>3 \mathrm{~cm}$ in diameter or signs of brainstem compression and/or hydrocephalus. ${ }^{16,17}$ All patients underwent craniotomy hematoma evacuation under general anesthesia. Surgery was only ended after ensuring that there was no active bleeding during the procedure. In patients with $\mathrm{SCH}$ that was associated with obvious acute hydrocephalus and/or a massive ventricular hematoma, ventricular drainage was performed simultaneously. During the operation, if the brain pressure was still high after the hematoma was evacuated, craniectomy was performed simultaneously.

\section{CT Scan and Imaging Analysis}

Cranial CT scans at 12 hours prior to initial surgery, within 24 hours, and 48-72 hours after initial surgery were performed in all patients. The patients also underwent a CT scan when their neurological state significantly deteriorated. The occurrence of PRB was recorded during the first 72 hours following the surgery. PRB was defined as a new postoperative hematoma. The appearance of the new hematoma was different than the appearance on the preoperative $\mathrm{CT}$ scan, and the volume of the new hematoma was more than half of the original hematoma volume. PRB should be distinguished from residual hematoma based on the intraoperative record and consistency of the hematoma appearance between the postoperative and preoperative CT scans. The appearance of the residual hematoma was consistent between the postoperative and preoperative CT scans (ie, the same shape and density) with no trace of the surgical procedure, while PRB displayed a different appearance between the postoperative and preoperative $\mathrm{CT}$ scans (Figure 1A-E). ${ }^{13,14}$

Hematoma volume was calculated as $\sum$ [Layer thickness $\times$ Area of each layer]. The area of each layer was measured using ImageJ software. ${ }^{18}$ The characteristics of SCH on CT scan include black hole sign, island sign, swirl sign, and mixed density. The black hole sign was defined as: (1) a black hole (relatively hypoattenuated area) encapsulated within the hyperattenuating hematoma; (2) the black hole was not connected with the adjacent brain tissue, and the shape could be round, oval, or rod-like; (3) an identifiable border from the relatively hypoattenuated area; and (4) at least a 28 Houns-field unit (HU) difference in density between the 2 regions (Figure 1F). ${ }^{19}$ The island sign was defined as: (1) equal to or more than 3 small separated hematomas, small hematomas could be round or oval and are not connected with the main hematoma; (2) equal to or more than 4 small hematomas, all or some of which may connect with the main hematoma, and small hematomas should be sprout-like or bubble-like but not lobulated (Figure 1G). ${ }^{20,21}$ The swirl sign was defined as a low-density area surrounded by a high-density hematoma (Figure $1 \mathrm{H}$ ). ${ }^{22,23}$ The mixed density was defined as a high-density and low-density hematoma irregularly mixed together (Figure 1I). Characteristics of the hematoma on CT scan were evaluated by 2 experienced neurosurgeons who were blinded to this study. 

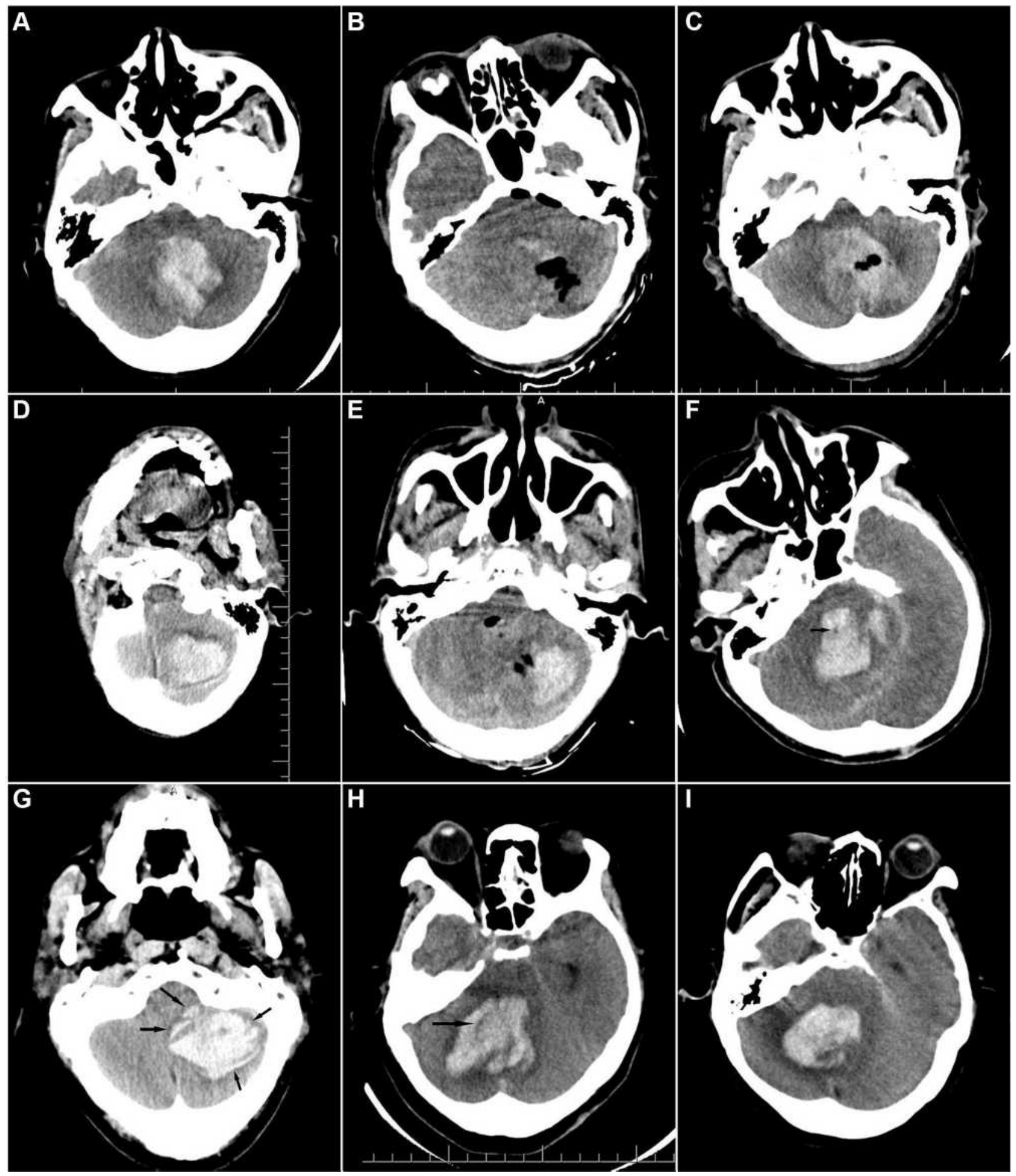

Figure I (A-C) Postoperative rebleeding; (D-E) hematoma residual; (F) black hole sign; (G) island sign; (H) swirl sign; (I) mixed density.

Parameters Included in the Statistical Analysis

The following clinical and radiological parameters were collected and included in the statistical analysis: age, sex, diabetes mellitus, smoking, daily alcohol consumption, antiplatelet use, systolic blood pressure (SBP) on arrival, Glasgow Coma Scale (GCS) score at admission, thrombocytopenia (defined as a platelet count less than $100 \times 10^{3}$ / $\mathrm{mL}$ ), coagulopathy (defined as a prothrombin time- 
international normalized ratio of $>1.4$ or an activated partial thromboplastin time of $\geq 40$ seconds.), early surgery (defined as surgery performed within 8 hours posthemorrhage), duration of surgery, hematoma volume, black hole sign, island sign, swirl sign, mixed density, and operation method (hematoma evacuation or hematoma evacuation + ventricular drainage). Short-term prognosis was assessed by using the modified Rankin Scale (mRS) at 3 months after the initial surgery, and a $\mathrm{mRS} \geq 4$ was defined as a poor outcome. ${ }^{24-26}$

\section{Statistical Analyses}

All statistical analyses were performed with SPSS (version 22.0, IBM Corp.). Data are presented as the numbers of patients (percentages) and the means $\pm \mathrm{SD}$ (standard deviation) for categorical and continuous variables, respectively. Predictors of PRB were first assessed using a univariable logistic regression model, and predictors with a $P$ value of less than 0.05 were then included in the multivariable logistic regression model to identify independent predictors. A receiver operating characteristic (ROC) curve was created based on independent risk factors obtained from the multivariable logistic analyses, and cutoff values of the continuous variables were recorded. The interobserver agreement for identifying the hematoma characteristics was determined using kappa values. A $P$ value of less than 0.05 was considered statistically significant.

\section{Results}

\section{Baseline Characteristics}

After screening, 62 patients who met the enrollment criteria were included in this study. The mean age of the patients at admission was $67.39 \pm 10.21$ years (age range, 36-87 years). There were 15 women and 47 men, and the ratio was 1:3.13. The PRB was observed in 13 patients (21\%). Eight patients died within three months after the initial operation, two patients who died before the postoperative CT examination were excluded from the statistical analyses, and the mortality rate was $12.5 \%(8 / 64)$. Poor prognosis was observed in 33 patients $(53.2 \%)$. The average hematoma volume was 25.19 $\pm 10.02 \mathrm{~mL}$. Other baseline clinical and radiological characteristics of the patients are listed in Table 1.

\section{Prevalence and Interobserver Agreement of Hematoma Characteristics}

Of the 62 patients, the black hole sign was identified in 9 patients $(14.5 \%)$, the island sign was identified in 6 patients $(9.7 \%)$, the swirl sign was identified in 9 patients
Table I Baseline, Clinical, and Radiographic Characteristics of Patients with $\mathrm{SCH}$

\begin{tabular}{|l|l|}
\hline Characteristic & Patients with SCH \\
\hline Age (years) & $67.39 \pm 10.21$ \\
Female Sex & $15(24.2)$ \\
Diabetes mellitus & $7(11.3)$ \\
Smoking & $10(16.1)$ \\
Daily alcohol consumption & $8(12.9)$ \\
Antiplatelet drug & $6(9.7)$ \\
SBP on arrival (mmHg) & $187.34 \pm 33.36$ \\
GCS score $\leq 8$ & $26(41.9)$ \\
Thrombocytopenia & $10(16.1)$ \\
Coagulopathy & $6(9.7)$ \\
Early surgery & $44(71)$ \\
Duration of surgery (minutes) & $240.19 \pm 61.13$ \\
Hematoma volume (mL) & $25.19 \pm 10.02$ \\
Black hole sign & $9(14.5)$ \\
Island sign & $6(9.7)$ \\
Swirl sign & $7(11.3)$ \\
Mixed density & $20(32.3)$ \\
Hydrocephalus & $14(22.6)$ \\
\hline Operation method & \\
HE & $22(35.5)$ \\
HE + VD & $40(64.5)$ \\
\hline
\end{tabular}

Abbreviations: GCS, Glasgow Coma Scale; HE, hematoma evacuation; SBP, systolic blood pressure; $\mathrm{SCH}$, spontaneous cerebellar hemorrhage; VD, ventricular drainage.

(14.5\%), and mixed density was identified in 20 patients $(32.3 \%)$. Interobserver agreement for the black hole sign, island sign, swirl sign, and mixed density was $\kappa=0.0 .689$, $\kappa=0.742, \kappa=0.772$, and $\kappa=0.730$, respectively (Table 2 ).

\section{Predictors for PRB in Patients with SCH}

As shown in Table 3, univariate logistic regression analysis revealed that hematoma volume $(\mathrm{OR}=29.333,95 \%$ $\mathrm{CI}=2.404-357.848, \quad P=0.008), \quad$ black hole sign $(\mathrm{OR}=7.031,95 \% \mathrm{CI}=1.546-31.977, P=0.012)$, and mixed density $(\mathrm{OR}=4.933,95 \% \mathrm{CI}=1.354-17.98, P=0.016)$ were associated with the $\mathrm{PRB}$ of $\mathrm{SCH}$. A multivariate analysis

Table 2 Radiological Characteristics and Interobserver Agreement ( $\mathrm{N}=62)$

\begin{tabular}{|l|c|c|c|}
\hline $\begin{array}{l}\text { Radiological } \\
\text { Characteristics }\end{array}$ & $\begin{array}{c}\text { Number of } \\
\text { Patients with } \\
\text { Positive Sign (\%) }\end{array}$ & p value & $\begin{array}{c}\text { Interobserver } \\
\text { Agreement } \\
\text { (к) }\end{array}$ \\
\hline Black hole sign & $9(14.5)$ & $<0.001$ & 0.689 \\
Island sign & $6(9.7)$ & $<0.001$ & 0.742 \\
Swirl sign & $7(11.3)$ & $<0.001$ & 0.772 \\
Mixed density & $20(32.3)$ & $<0.001$ & 0.730 \\
\hline
\end{tabular}


Table 3 Logistic Regression Analysis of Predictors for PRB in Patients with $\mathrm{SCH}(\mathrm{N}=13)$

\begin{tabular}{|c|c|c|c|c|c|c|}
\hline \multirow[t]{2}{*}{ Predictors } & \multicolumn{3}{|c|}{ Crude Model } & \multicolumn{3}{|c|}{ Multivariable Model } \\
\hline & OR & $95 \% \mathrm{Cl}$ & p value & OR & $95 \% \mathrm{Cl}$ & p value \\
\hline Age (per 10-yr increase) & 1.200 & $0.147-9.768$ & 0.865 & & & \\
\hline Female Sex & 0.208 & $0.025-1.757$ & 0.149 & & & \\
\hline Diabetes mellitus & 1.600 & $0.273-9.374$ & 0.602 & & & \\
\hline Smoking & 1.800 & $0.394-8.215$ & 0.448 & & & \\
\hline Daily alcohol consumption & 2.640 & $0.540-12.914$ & 0.231 & & & \\
\hline Antiplatelet drug & 2.045 & $0.331-12.636$ & $0.44 I$ & & & \\
\hline SBP on arrival (per 20-mmHg increase) & 1.833 & $0.318-10.573$ & 0.498 & & & \\
\hline GCS score $\leq 8$ & 2.756 & $0.782-9.708$ & 0.115 & & & \\
\hline Thrombocytopenia & 0.932 & $0.173-5.032$ & 0.935 & & & \\
\hline Coagulopathy & 0.733 & $0.078-6.888$ & 0.786 & & & \\
\hline Early surgery & $1.47 \mid$ & $0.353-6.121$ & 0.596 & & & \\
\hline Duration of surgery (per I-hr increase) & 0.667 & $0.057-7.852$ & 0.747 & & & \\
\hline Hematoma volume (per 10-mL increase) & 29.333 & $2.404-357.848$ & 0.008 & 15.135 & $1.075-213.101$ & 0.044 \\
\hline Black hole sign & 7.031 & $1.546-31.977$ & 0.012 & 4.087 & $0.674-24.791$ & 0.126 \\
\hline Island sign & 2.045 & $0.331-12.636$ & $0.44 I$ & & & \\
\hline Swirl sign & 2.150 & $0.458-10.103$ & 0.332 & & & \\
\hline Mixed density & 4.933 & $1.354-17.980$ & 0.016 & 3.732 & $0.834-16.701$ & 0.085 \\
\hline Hydrocephalus & 1.036 & $0.242-4.437$ & 0.962 & & & \\
\hline Operation method (HE + VD) & 1.176 & $0.333-4.159$ & 0.801 & & & \\
\hline
\end{tabular}

Abbreviations: $\mathrm{Cl}$, confidence interval; GCS, Glasgow Coma Scale; HE, hematoma evacuation; OR, odds ratio; SBP, systolic blood pressure; SCH, spontaneous cerebellar hemorrhage; VD, ventricular drainage.

involving these three variables obtained in the univariate analyses was performed. Hematoma volume was the only independent predictor of $\mathrm{PRB} \quad(\mathrm{OR}=15.135,95 \%$ $\mathrm{CI}=1.075-213.101, P=0.044)$. Next, an ROC analysis of the hematoma volume was performed. The sensitivity and specificity of hematoma volume to PRB were $61.5 \%$ and $83.7 \%$, respectively. The area under the curve (AUC) was $0.747,95 \% \mathrm{CI}=0.621-0.849$. The cutoff value of hematoma was $>29.3 \mathrm{~mL}$, suggesting that $\mathrm{SCH}$ patients with hematoma volumes greater than $29.3 \mathrm{~mL}$ are prone to $\mathrm{PRB}$ (Figure 2).

\section{Predictors of Short-Term Poor Prognosis in Patients with $\mathrm{SCH}$}

In univariate logistic regression analysis, GCS score $\leq 8$ $(\mathrm{OR}=12.5,95 \% \mathrm{CI}=3.476-44.948, P<0.001)$, early surgery $(\mathrm{OR}=3.176,95 \% \mathrm{CI}=1.003-10.059, P=0.049)$, hematoma volume $(\mathrm{OR}=17,95 \% \mathrm{CI}=1.683-171.703, P=0.016)$, and $\mathrm{PRB}(\mathrm{OR}=16,95 \% \mathrm{CI}=1.926-132.899, P=0.01)$ were related to poor prognosis of patients with $\mathrm{SCH}$. Because the $P$ value of these four variables was less than 0.05 , we next included them in the multivariable logistic regression model. The results revealed that GCS score $\leq 8$ $(\mathrm{OR}=5.131,95 \% \mathrm{CI}=1.030-25.554, P=0.046)$ and $\mathrm{PRB}$
$(\mathrm{OR}=13.17,95 \% \mathrm{CI}=1.316-131.798, P=0.028)$ were independent risk factors for poor prognosis (Table 4). To assess the efficiency of the GCS score and PRB in predicting poor prognosis, we further conducted an ROC analysis on these variables. The sensitivity and specificity of the GCS

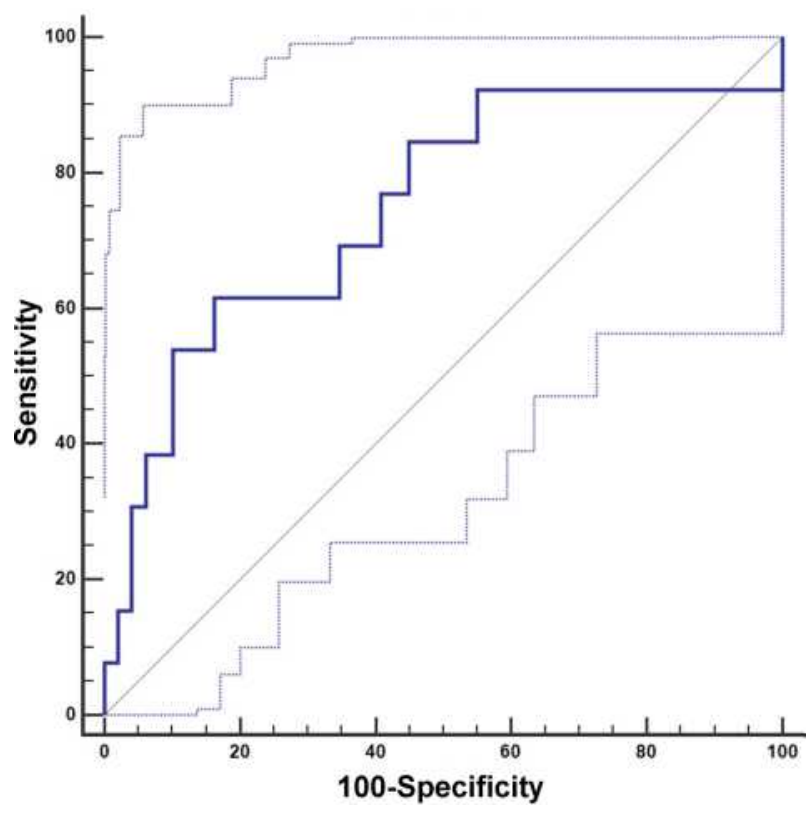

Figure 2 ROC analysis of the hematoma volume to postoperative rebleeding. 
Table 4 Logistic Regression Analysis of Predictors for Poor Prognosis in Patients with SCH (N=33)

\begin{tabular}{|c|c|c|c|c|c|c|}
\hline \multirow[t]{2}{*}{ Predictors } & \multicolumn{3}{|c|}{ Crude Model } & \multicolumn{3}{|c|}{ Multivariable Model } \\
\hline & OR & $95 \% \mathrm{Cl}$ & p value & OR & $95 \% \mathrm{Cl}$ & p value \\
\hline Age (per 10-yr increase) & 2.222 & $0.192-25.723$ & 0.523 & & & \\
\hline Female Sex & 1.437 & $0.44 I-4.682$ & 0.547 & & & \\
\hline Diabetes mellitus & 0.310 & $0.055-1.737$ & 0.183 & & & \\
\hline Smoking & 0.857 & $0.221-3.320$ & 0.823 & & & \\
\hline Daily alcohol consumption & 1.548 & $0.336-7.130$ & 0.575 & & & \\
\hline Antiplatelet use & 1.862 & $0.315-11.003$ & 0.493 & & & \\
\hline SBP on arrival (per 20-mmHg increase) & 2.000 & $0.407-9.838$ & 0.394 & & & \\
\hline GCS score $\leq 8$ & 12.500 & $3.476-44.948$ & $<0.001$ & 5.131 & $1.030-25.554$ & 0.046 \\
\hline Thrombocytopenia & 4.320 & $0.836-22.320$ & 0.081 & & & \\
\hline Coagulopathy & 0.403 & $0.068-2.385$ & 0.317 & & & \\
\hline Early surgery & 3.176 & $1.003-10.059$ & 0.049 & 1.237 & $0.263-5.821$ & 0.788 \\
\hline Duration of surgery (per I-hr increase) & 1.524 & $0.250-9.295$ & 0.648 & & & \\
\hline Hematoma volume & 17.000 & $1.683-171.703$ & 0.016 & 0.216 & $0.014-3.229$ & 0.267 \\
\hline Black hole sign & 3.635 & $0.690-19.138$ & 0.128 & & & \\
\hline Island sign & 5.000 & $0.548-45.579$ & 0.153 & & & \\
\hline Swirl sign & 3.647 & $0.576-19.033$ & 0.133 & & & \\
\hline Mixed density & 2.043 & $0.680-6.140$ & 0.203 & & & \\
\hline Hydrocephalus & 1.800 & $0.52-6.164$ & 0.349 & & & \\
\hline Operation method (HE + VD) & 0.343 & $0.117-1.009$ & 0.052 & & & \\
\hline PRB & 16.000 & $1.926-132.899$ & 0.010 & 13.170 & $1.316-131.798$ & 0.028 \\
\hline
\end{tabular}

Abbreviations: $\mathrm{Cl}$, confidence interval; GCS, Glasgow Coma Scale; HE, hematoma evacuation; OR, odds ratio; PRB, postoperative rebleeding; SBP, systolic blood pressure; $\mathrm{SCH}$, spontaneous cerebellar hemorrhage; VD, ventricular drainage.

score to predict poor prognosis were $86.21 \%$ and $66.67 \%$, respectively, and the AUC was 0.764 , 95\% $\mathrm{CI}=0.639-0.863$. The sensitivity and specificity of PRB to poor prognosis were $36.4 \%$ and $96.6 \%$, respectively, and the AUC was $0.67,95 \% \mathrm{CI}=0.533-0.780$ (Figure 3).

\section{Discussion}

This study investigated whether clinical and radiographic parameters could predict PRB and short-term prognosis of $\mathrm{SCH}$. The results proved that hematoma volume was the only independent risk factor for PRB. The likely reason is that a large volume of hematoma has a large surgical field, which increases the risk of rebleeding. GCS score at admission and PRB were independent risk factors for short-term poor prognosis of SCH. Other factors had no significant correlation with either PRB or short-term prognosis.

The incidence of PRB was $21 \%$, higher than the previously reported $7.2-10.9 \% .^{13-15}$ One reason is that the incidence of PRB in the cerebellum is higher, and previous reports did not distinguish between supratentorial hematoma and cerebellar hematoma. Another reason is that the definition of PRB can differ. We defined it as a new postoperative hematoma, and the volume of the new hematoma was more than half of the original hematoma volume. Other authors define PRB as having a mass effect

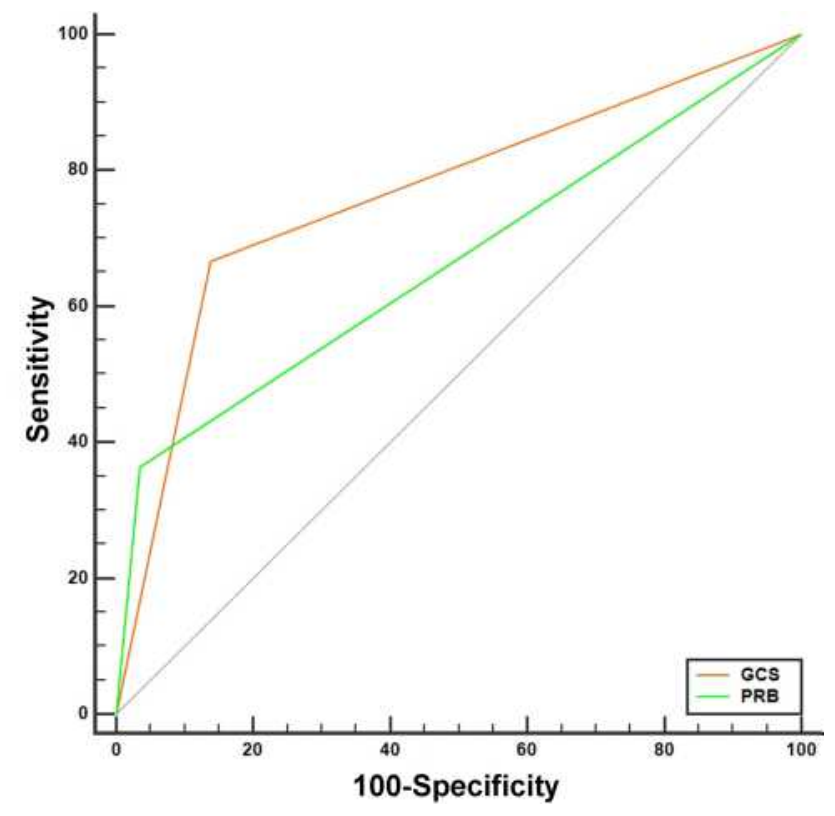

Figure 3 ROC analysis of the GCS and postoperative rebleeding to short-term prognosis. 
that causes neurological deterioration. If the new hematoma was just within the removed hematoma cavity but had no mass effect, it was not considered a PRB. ${ }^{13,14}$ However, this definition of PRB may not apply to the cerebellum because most patients with cerebellar ICH undergo suboccipital decompression and/or ventricular drainage at the same time as surgery; thus, even if the volume of the new hematoma is the same as the original hematoma, it may not cause neurological deterioration.

CT angiography spot signs have been well documented in ICH and can predict hematoma expansion and active bleeding during surgery. ${ }^{27-29}$ In addition, the spot sign has also been shown to predict rebleeding after ICH surgery, ${ }^{14,15}$ and it is a risk factor for in-hospital mortality and poor outcome among survivors. ${ }^{15,24,27}$ However, spot signs require immediate admission for $\mathrm{CT}$ angiography examination within the initial several hours after the onset of symptoms, which is not applicable in some institutions. It is also not suitable for patients who have formed a brain herniation when admitted and need emergency surgery to relieve brainstem compression. In addition, $\mathrm{CT}$ angiography examination requires contrast administration, which is contraindicated in patients with renal function impairment. In this cohort, CT angiography was performed in 23 patients, and spot signs were identified in only one patient without PRB; therefore, we did not include them in the statistical analysis. Further studies are needed to confirm whether spot signs are related to the $\mathrm{PRB}$ of $\mathrm{SCH}$.

Recently, some noncontrast CT markers, including the black hole sign, island sign, swirl sign, and blend sign (defined as blending of hyperattenuating area and hypoattenuating region with a well-defined margin ${ }^{14}$ ), have been reported to predict the hematoma expansion of ICH under conservative treatment. ${ }^{13,20,21,23,30-32}$ Among these radiological characteristics, island sign has been reported to predict short-term and long-term poor prognoses and mortality in ICH patients, ${ }^{20,21,30-32}$ and the blend sign is likely to be an independent risk factor for PRB in ICH patients. ${ }^{13}$ However, in this study, these radiological characteristics had no significant correlation with either PRB or shortterm prognosis of patients with $\mathrm{SCH}$. In addition, the blend sign was only observed in one patient, and we excluded it from the statistical analysis.

In the present study, thrombocytopenia, coagulopathy, and the use of antiplatelets were not associated with either PRB or short-term prognosis. This is consistent with the results reported in prior studies. ${ }^{13,14,25}$ Early surgery within 4 hours of symptom onset is a reported risk factor for PRB. ${ }^{12}$ In contrast, a meta-analysis performed by Gregson BA indicated that early surgery within 8 hours post-hemorrhage can improve the prognosis of $\mathrm{ICH}$ patients. ${ }^{33}$ However, we and others have demonstrated that the timing of surgery is not correlated with either PRB or prognosis. ${ }^{13,14}$

This study confirmed that PRB is a risk factor for poor prognosis in patients with $\mathrm{SCH} .{ }^{12}$ GCS score at admission was another independent risk factor for short-term poor prognosis in this study. The GCS score at admission is widely accepted as an independent risk factor for both short- and long-term poor prognosis in patients with $\mathrm{SCH}$. Al Safatli D showed that a GCS score $<10$ on admission was associated with increased 30-day mortality and poorer short-term outcomes of patients with $\mathrm{SCH} .{ }^{34}$ Other researchers have demonstrated that GCS scores $\leq 8$ on arrival are a strong predictive factor for first-week mortality and shortand long-term poor prognoses of patients with $\mathrm{SCH}^{35-38}$ Hydrocephalus was observed in $22.6 \%$ of all patients, similar to the previously reported $23 \%{ }^{39}$ Additionally, hydrocephalus and age have been indicated to be predictors for poor outcomes of SCH patients, ${ }^{38,40,41}$ and the results from this study and others revealed that they were not related to short-term poor prognosis. ${ }^{34,37}$

This retrospective cohort study had some limitations. First, the study was limited by the small sample size due to the scarcity of patients with $\mathrm{SCH}$, and a larger number of patients from multiple centers is needed to verify our conclusions. Second, different neurosurgeons performed different surgical procedures, affecting whether suboccipital decompression is required and when to remove the drainage tube. These details were not captured in this study. Third, CT angiography was only performed in 23 patients; therefore, we are not sure whether the spot sign was related to PRB and poor short-term prognosis. Finally, most patients with $\mathrm{SCH}$ have consciousness disturbances when they are admitted to the hospital. The time of onset and accompanying diseases stated by family members may have led to bias.

\section{Conclusions}

Hematoma volume is likely to be a strong predictor of PRB among patients with SCH. Neurosurgeons should carefully control bleeding in the operative field when surgically treating $\mathrm{SCH}$ in patients with large hematomas. GCS scores $\leq 8$ on arrival and PRB were significant predictors of short-term poor outcome. For patients with 
a GCS score $\leq 8$ on admission and/or PRB, it is important to weigh the benefit of survival against the high risk of poor quality of life and significant functional disability that may be achieved by extended or intensified therapy.

\section{Abbreviations}

AUC, area under the curve; CI, confidence interval; GCS, Glasgow Coma Scale; HE, ICH, intracerebral hemorrhage; mRS, modified Rankin Scale; PRB, postoperative rebleeding; ROC, receiver operating characteristic; SBP, systolic blood pressure; $\mathrm{SCH}$, spontaneous cerebellar hemorrhage; S.D, standard deviation.

\section{Ethics Statement}

We obtained ethical approval to carry out this study from the Institutional Review Board (IRB) of The First Affiliated Hospital (YiJiShan Hospital) of Wannan Medical College, Wuhu, China. All patients or their relatives provided written informed consent for this study, which was carried out in compliance with the Helsinki Declaration. All individual information was strictly kept confidential and anonymous in the manuscript.

\section{Acknowledgment}

This study was supported by grants from Anhui Collegiate Major Natural Science Research Projects (Grant No. KJ2017A267, KJ2018ZD027), Nature Science Research Project of Anhui province (Grant No.1908085QH356), Three new projects of Wannan Medical College (Y20082), and Natural Science Research Project of Wannan Medical College (KY24790417).

\section{Disclosure}

The authors report no conflicts of interest in this study.

\section{References}

1. Cordonnier C, Demchuk A, Ziai W, et al. Intracerebral haemorrhage: current approaches to acute management. Lancet. 2018;392 (10154):1257-1268. doi:10.1016/S0140-6736(18)31878-6

2. Chen S, Zeng L, Hu Z. Progressing haemorrhagic stroke: categories, causes, mechanisms and managements. $J$ Neurol. 2014;261 (11):2061-2078. doi:10.1007/s00415-014-7291-1

3. Qureshi A, Mendelow AD, Hanley DF. Intracerebral haemorrhage. Lancet. 2009;373(9675):1632-1644. doi:10.1016/S0140-6736(09) 60371-8

4. Flaherty ML, Woo D, Haverbusch M, et al. Racial variations in location and risk of intracerebral hemorrhage. Stroke. 2005;36 (5):934-947. doi:10.1161/01.STR.0000160756.72109.95

5. Dolderer S, Kallenberg K, Aschoff A, et al. Long-term outcome after spontaneous cerebellar haemorrhage. Eur Neurol. 2004;52 (2):112-119. doi:10.1159/000080268
6. Keep RF, Hua Y, Xi G. Intracerebral haemorrhage: mechanisms of injury and therapeutic targets. Lancet Neurol. 2012;11(8):720-731. doi:10.1016/S1474-4422(12)70104-7

7. Hackenberg KA, Unterberg AW, Jung CS, et al. Does suboccipital decompression and evacuation of intraparenchymal hematoma improve neurological outcome in patients with spontaneous cerebellar hemorrhage? Clin Neurol Neurosurg. 2017;155:22-29. doi:10.1016/j.clineuro.2017.01.019

8. Mendelow AD, Gregson BA, Rowan EN, et al. Early surgery versus initial conservative treatment in patients with spontaneous supratentorial lobar intracerebral haematomas (STICH II): a randomised trial. Lancet. 2013;382(9890):397-408. doi:10.1016/S0140-6736(13) 60986-1

9. de Oliveira Manoel AL. Surgery for spontaneous intracerebral hemorrhage. Crit Care. 2020;24(1):45. doi:10.1186/s13054-020$2749-2$

10. Kuramatsu JB, Biffi A, Gerner ST, et al. Association of surgical hematoma evacuation vs conservative treatment with functional outcome in patients with cerebellar intracerebral hemorrhage. JAMA. 2019;322(14):1392-1403. doi:10.1001/jama.2019.13014

11. Singh SD, Brouwers HB, Senff JR, et al. Haematoma evacuation in cerebellar intracerebral haemorrhage: systematic review. $J$ Neurol Neurosurg Psychiatry. 2020;91(1):82-87. doi:10.1136/jnnp-2019321461

12. Morgenstern LB, Demchuk AM, Kim DH, et al. Rebleeding leads to poor outcome in ultra-early craniotomy for intracerebral hemorrhage. Neurology. 2001;56(10):1294-1299. doi:10.1212/wnl.56.10.1294

13. Yagi K, Tao Y, Hara K, et al. Does noncontrast computed tomography scan predict rebleeding after endoscopic surgery for spontaneous intracerebral hemorrhage? World Neurosurg. 2019;127:e965-e971. doi:10.1016/j.wneu.2019.04.010

14. Miki K, Yagi K, Nonaka M, et al. Spot sign as a predictor of rebleeding after endoscopic surgery for intracerebral hemorrhage. J Neurosurg. 2018:1-6. doi:10.3171/2017.12.JNS172335

15. Miki K, Abe H, Nonaka M, et al. Impact of spot sign etiology in supratentorial intracerebral hemorrhage on outcomes of endoscopic surgery. World Neurosurg. 2020;133:e281-e287. doi:10.1016/j. wneu.2019.08.244

16. de Oliveira Manoel AL, Goffi A, Zampieri FG, et al. The critical care management of spontaneous intracranial hemorrhage: a contemporary review. Crit Care. 2016;20(1):272. doi:10.1186/s13054-016-1432-0

17. Hemphill JC 3rd, Greenberg SM, Anderson CS, et al. Guidelines for the management of spontaneous intracerebral hemorrhage: a guideline for healthcare professionals from the American heart association/American stroke association. Stroke. 2015;46 (7):2032-2060. doi:10.1161/STR.0000000000000069

18. Shen J, Xin W, Li Q, et al. A grading system for the prediction of unilateral chronic subdural hematoma recurrence after initial single burr hole evacuation. Risk Manag Healthc Policy. 2019;12:179-188. doi:10.2147/RMHP.S222144

19. Li Q, Zhang G, Xiong X, et al. Black hole sign: novel imaging marker that predicts hematoma growth in patients with intracerebral hemorrhage. Stroke. 2016;47(7):1777-1781. doi:10.1161/ STROKEAHA.116.013186

20. Li Q, Liu QJ, Yang WS, et al. Island sign: an imaging predictor for early hematoma expansion and poor outcome in patients with intracerebral hemorrhage. Stroke. 2017;48(11):3019-3025. doi:10.1161/ STROKEAHA.117.017985

21. Zhang F, Li H, Qian J, et al. Island sign predicts long-term poor outcome and mortality in patients with intracerebral hemorrhage. World Neurosurg. 2018;120:e304-e312. doi:10.1016/j.wneu.20 18.08 .056

22. Yu Z, Zheng J, He M, et al. Accuracy of swirl sign for predicting hematoma enlargement in intracerebral hemorrhage: a meta-analysis. J Neurol Sci. 2019;399:155-160. doi:10.1016/j.jns.2019.02.032 
23. Ng D, Churilov L, Mitchell P, et al. The CT swirl sign is associated with hematoma expansion in intracerebral hemorrhage. AJNR Am $J$ Neuroradiol. 2018;39(2):232-237. doi:10.3174/ajnr.A5465

24. Delgado Almandoz JE, Yoo AJ, Stone MJ, et al. The spot sign score in primary intracerebral hemorrhage identifies patients at highest risk of in-hospital mortality and poor outcome among survivors. Stroke. 2010;41(1):54-60. doi:10.1161/STROKEAHA.109.565382

25. Song X, Zhang Q, Cao Y, et al. Antiplatelet therapy does not increase mortality of surgical treatment for spontaneous intracerebral haemorrhage. Clin Neurol Neurosurg. 2020;196:105873. doi:10.1016/j.clineuro.2020.105873

26. Troberg E, Kronvall E, Hansen BM, et al. Prediction of long-term outcome after intracerebral hemorrhage surgery. World Neurosurg. 2018:S1878-8750. doi:10.1016/j.wneu.2018.12.012

27. Demchuk AM, Dowlatshahi D, Rodriguez-Luna D, et al. Prediction of haematoma growth and outcome in patients with intracerebral haemorrhage using the CT-angiography spot sign (PREDICT): a Prospective Observational Study. Lancet Neurol. 2012;11 (4):307-314. doi:10.1016/S1474-4422(12)70038-8

28. Dowlatshahi D, Wasserman JK, Momoli F, et al. Evolution of computed tomography angiography spot sign is consistent with a site of active hemorrhage in acute intracerebral hemorrhage. Stroke. 2014;45 (1):277-280. doi:10.1161/STROKEAHA.113.003387

29. Brouwers HB, Raffeld MR, van Nieuwenhuizen KM, et al. CT angiography spot sign in intracerebral hemorrhage predicts active bleeding during surgery. Neurology. 2014;83(10):883-889. doi:10.1212/WNL.0000000000000747

30. Zhang F, Zhang S, Tao C, et al. The comparative study of island sign and the spot sign in predicting short-term prognosis of patients with intracerebral hemorrhage. $J$ Neurol Sci. 2019;396:133-139. doi:10.1016/j.jns.2018.11.022

31. Deng L, Zhang G, Wei X, et al. Comparison of satellite sign and island sign in predicting hematoma growth and poor outcome in patients with primary intracerebral hemorrhage. World Neurosurg. 2019;127:e818-e825. doi:10.1016/j.wneu.2019.03.273

32. Zheng J, Yu Z, Wang C, et al. Evaluating the predictive value of island sign and spot sign for hematoma expansion in spontaneous intracerebral hemorrhage. World Neurosurg. 2018;117:e167-e171. doi:10.1016/j.wneu.2018.05.221
33. Gregson BA, Broderick JP, Auer LM, et al. Individual patient data subgroup meta-analysis of surgery for spontaneous supratentorial intracerebral hemorrhage. Stroke. 2012;43(6):1496-1504. doi:10.1161/STROKEAHA.111.640284

34. Al Safatli D, Guenther A, McLean AL, et al. Prediction of 30-day mortality in spontaneous cerebellar hemorrhage. Surg Neurol Int. 2017;8(1):282. doi:10.4103/sni.sni_479_16

35. Wu YT, Li TY, Chiang SL, et al. Predictors of first-week mortality in patients with acute spontaneous cerebellar hemorrhage. Cerebellum. 2013;12(2):165-170. doi:10.1007/s12311-012-0410-6

36. Satopää J, Meretoja A, Koivunen RJ, et al. Treatment of intracerebellar haemorrhage: poor outcome and high long-term mortality. Surg Neurol Int. 2017;8(1):272. doi:10.4103/sni.sni_168_17

37. Pong V, Chan $\mathrm{KH}$, Chong $\mathrm{BH}$, et al. Long-term outcome and prognostic factors after spontaneous cerebellar hemorrhage. Cerebellum. 2012;11(4):939-945. doi:10.1007/s12311-012-0371-9

38. Matsukawa H, Shinoda M, Fujii M, et al. Relationships among hematoma diameter, location categorized by vascular territory, and 1-year outcome in patients with cerebellar hemorrhage. World Neurosurg. 2012;77(3-4):507-511. doi:10.1016/j.wneu.2011.05.018

39. Bhattathiri PS, Gregson B, Prasad KS, et al. Intraventricular hemorrhage and hydrocephalus after spontaneous intracerebral hemorrhage: results from the STICH trial. Acta Neurochir Suppl. 2006;96:65-68. doi:10.1007/3-211-30714-1_16

40. Tsitsopoulos PP, Tobieson L, Enblad P, et al. Prognostic factors and long-term outcome following surgical treatment of 76 patients with spontaneous cerebellar haematoma. Acta Neurochir (Wien). 2012;154 (7):1189-1195. doi:10.1007/s00701-012-1372-7

41. Dahdaleh NS, Dlouhy BJ, Viljoen SV, et al. Clinical and radiographic predictors of neurological outcome following posterior fossa decompression for spontaneous cerebellar hemorrhage. J Clin Neurosci. 2012;19(9):1236-1241. doi:10.1016/j.jocn.2011.11.025
Risk Management and Healthcare Policy

\section{Publish your work in this journal}

Risk Management and Healthcare Policy is an international, peerreviewed, open access journal focusing on all aspects of public health, policy, and preventative measures to promote good health and improve morbidity and mortality in the population. The journal welcomes submitted papers covering original research, basic science, clinical \& epidemiological studies, reviews and evaluations, guidelines, expert opinion and commentary, case reports and extended reports. The manuscript management system is completely online and includes a very quick and fair peer-review system, which is all easy to use. Visit http://www.dovepress.com/testimonials.php to read real quotes from published authors. 